\title{
Comparative study of the osteogenic differentiation capacity of human bone marrow- and human adipose-derived stem cells under cyclic tensile stretch using quantitative analysis
}

\author{
YAPING YE*, YU DU*, FENGJING GUO, CHEN GONG, KAIXIANG YANG and LIANG QIN \\ Department of Orthopedics, Tongji Hospital, Tongji Medical College, Huazhong University \\ of Science and Technology, Wuhan, Hubei, P.R. China
}

Received June 18, 2012; Accepted August 2, 2012

DOI: 10.3892/ijmm.2012.1123

\begin{abstract}
Studies comparing the osteogenic differentiation capacity of human bone marrow-derived stem cells (hBMSCs) and human adipose-derived stem cells (hASCs) cultured in osteogenic differentiation medium have been inconclusive. Apart from chemical stimuli, mechanical stimuli have also been shown to be important in bone tissue engineering, which is referred to as functional bone tissue engineering. hBMSCs and hASCs have been shown to be sensitive to both chemical and mechanical stimuli. In an attempt to find a better seed cell in functional bone tissue engineering, we tried to quantify the osteogenic differentiation capacity of hBMSCs and hASCs under both mechanical and chemical stimuli. In this study, hBMSCs and hASCs were isolated from the same volunteers. Cells were cultured in osteogenic differentiation medium with and without exposure to cyclic tensile stretch (CTS). Quantitative measurement of alkaline phosphatase (ALP) activity revealed that the osteogenic differentiation capacity of hBMSCs was similar to that of hASCs in the early phase of differentiation in the CTS-stimulated groups. Quantitative measurement of mineralization showed that the late-phase osteogenic differentiation capacity of the hBMSCs was superior to that of hASCs in the CTS-stimulated groups. Reverse transcription-polymerase chain reaction (RT-PCR) analysis was performed 5 and 10 days after cell culture. The results of the RT-PCR revealed that the osteogenic differentiation capacity of hASCs was inferior to that of hBMSCs both in the CTS-stimulated and unstimulated groups. All the results
\end{abstract}

Correspondence to: Dr Fengjing Guo, Department of Orthopedics, Tongji Hospital, Tongji Medical College, Huazhong University of Science and Technology, 1095 JieFang Avenue, Wuhan, Hubei 430030, P.R. China

E-mail: guofjdoc@163.com

*Contributed equally

Key words: osteogenic differentiation, bone marrow-derived stem cells, adipose-derived stem cells, cyclic tensile stretch showed that both hBMSCs and hASCs were sensitive to CTS during the osteogenic differentiation process. This study compared the osteogenic differentiation capacity of hBMSCs and hASCs in response to mechanical stimulations and has important implications for the use of stem cells in functional bone tissue engineering and regenerative medicine.

\section{Introduction}

Bone marrow-derived stem cells (BMSCs) have been widely utilized in bone tissue engineering $(1,2)$. They have been shown to readily attach to culture plates and differentiate down an osteogenic pathway under appropriate chemical stimulation (3). However, the aspiration of bone marrow is quite painful and the concentration of harvested cells is often low. These shortcomings have limited their application in bone tissue engineering.

More recently, adipose-derived stem cells (ASCs) have garnered great attention from researchers (4-7). These cells can be isolated by simple liposuction, and the harvested cell number is relatively high. Studies concentrating on ASCs have demonstrated their multi-differentiation potential. With the appropriate chemical stimulus, they can differentiate along an osteogenic lineage, a chondrogenic lineage, and an adipogenic lineage $(6,7)$.

Many studies have compared the osteogenic differentiation capacity of BMSCs and ASCs in an attempt to determine whether ASCs could be an alternative for BMSCs in bone tissue engineering, and thus far, the results have been inconsistent. De Ugarte et al (8) reported that there was no difference between BMSCs and ASCs in their osteogenic differentiation potential. Similarly, Hattori et al (9) showed in their study that ASCs are similar to BMSCs in their ability to differentiate into osteoblasts. However, Im et al (10) demonstrated that the level of mineralization and alkaline phosphatase (ALP) activity of BMSCs were 2- to 3-fold higher than that of ASCs when undergoing osteogenesis. In a recent study Vishnubalaji et al (11) concluded that the osteogenic differentiation potential of BMSCs was higher than that of ASCs when studied by qualitative methods, but when studied by the quantitative real-time polymerase chain reaction, there was no difference between them. 
Apart from sensitivity to chemical stimuli, BMSCs and ASCs were also found to be sensitive to mechanical stimuli (12-14). Numerous studies have shown that mechanical stimuli play an important role in bone tissue engineering, which is also referred to as functional bone tissue engineering $(15,16)$. In an attempt to find a better seed cell in functional bone tissue engineering, we tried to quantify the osteogenic differentiation capacity of BMSCs and ASCs in response to both chemical and mechanical stimuli.

In the present study, human bone marrow-derived stem cells (hBMSCs) and human adipose-derived stem cells (hASCs) were isolated from the same volunteers to eliminate differences caused by age and gender. Cells were then studied for their multi-differentiation ability and surface antigen expression profiles. Isolated hBMSCs and hASCs were cultured in osteogenic differentiation medium under cyclic tensile stretch (CTS) and static controls. Results revealed that both hBMSCs and hASCs were sensitive to CTS during the osteogenic differentiation process. Quantitative measurement of ALP activity showed that the early-phase osteogenic differentiation capacity of hBMSCs was similar to hASCs in the CTS-stimulated groups. While quantitative measurement of mineralization revealed that the late-phase osteogenic differentiation capacity of hBMSCs was superior to that of hASCs with statistical difference. RT-PCR revealed that the osteogenic differentiation capacity of hBMSCs were superior to that of hASCs both in the CTS-stimulated and unstimulated groups. This study compared the osteogenic differentiation capacity of hBMSCs and hASCs both in the CTS-stimulated and unstimulated groups and has great implication in the field of functional bone tissue engineering.

\section{Materials and methods}

Isolation and culture of hBMSCs and hASCs. Bone marrow and subcutaneous adipose tissue were obtained from the same healthy volunteers to exclude effects of age and gender. The surgical procedure was performed after informed consent was obtained from the volunteers (6 volunteers, 24-44 years of age). All procedures were approved by the Ethics Committee of Tongji Medical College, Huazhong University of Science and Technology, China. In brief, bone marrow aspirates were isolated by centrifugation at $1,200 \mathrm{x} \mathrm{g}$ for $20 \mathrm{~min}$, and mononucleated cells were then collected and suspended in growth medium which contained low glucose-Dulbecco's modified Eagle's medium (DMEM), 10\% fetal bovine serum (FBS), $2 \mathrm{mM}$ L-glutamine and 100 units penicillin-streptomycin. HASCs were isolated by density and differentiation adhesions. The adipose tissue was digested with $0.075 \%$ type I collagenase for $20 \mathrm{~min}$, and the hASCs-rich fraction was pelleted by centrifugation at $1,800 \mathrm{x}$ g for $20 \mathrm{~min}$. Red blood cells were lysed and cell pellets were suspended. Cells were then pelleted again by centrifugation at $1,800 \mathrm{x}$ g for $20 \mathrm{~min}$ and suspended in growth medium. Twenty-four hours later, non-adherent cells were washed with phosphate-buffered saline (PBS).

Finally, hBMSCs and hASCs were seeded at a density of $4 \times 10^{5}$ cells $/ \mathrm{ml}$ in $5 \% \mathrm{CO}_{2}$ and a water-saturated atmosphere and passaged at a 1:2 dilution once they had reached complete confluence. Cells that had passaged 3-7 times were used in this study.
Multi-differentiation ability tests. Cells were cultured in the growth medium for $24 \mathrm{~h}$ before changing into the corresponding adipogenic differentiation medium and osteogenic differentiation medium. The adipogenic differentiation medium contained DMEM supplemented with $10 \%$ FBS, $1 \mathrm{mM}$ dexamethasone (Sigma), $0.5 \mathrm{mM}$ methylisobutyl-xanthine (Sigma), $10 \mathrm{mg} / \mathrm{ml}$ insulin (Invitrogen Life Technologies, Carlsbad, CA) and $100 \mathrm{mM}$ indomethacin (Sigma). The osteogenic differentiation medium contained DMEM supplemented with $10 \%$ FBS, $10 \mathrm{mM}$ $\beta$-glycerophosphate (Sigma), $100 \mathrm{nM}$ dexamethasone and $0.1 \mathrm{mM}$ ascorbate-2-phosphate (Sigma). Both adipogenesis and osteogenesis lasted for a total of 2 weeks. Adipogenesis was evaluated by oil red $\mathrm{O}$ staining and osteogenesis was evaluated after staining with alizarin red.

Surface antigen characterization. Surface antigen expression profiles of hBMSCs and hASCs at passage 4 were examined using a flow cytometer, and isotype-matched normal $\operatorname{IgG}$ was used as the control. Fluorescein isothiocyanate (FITC)labeled anti-human CD44, CD45, CD105, phycoerythrin (PE)-labeled anti-human CD29 antibodies were purchased from BD Biosciences. After staining, the cells were washed twice in PBS containing 2\% FBS and analyzed using a Coulter FC500 flow cytometer.

Application of CTS. The 4-point bending mechanical stimulation device was purchased from Sichuan University at the requests of the authors. The diagrammatic sketch of the device is shown in Fig. 1A. The device consisted of 3 main parts: the main engine, the actuator and the cell culture Petri dish (Fig. 1B) and the main working process is shown in Fig. 1C. The frequency of the mechanical stimulation was $1 \mathrm{~Hz}$, with $15 \mathrm{sec}$ of rest after every 5 -sec working duration, with a total time of $2 \mathrm{~h}$ every day. The strain magnitude ranged from 0 to $3,200 \mu \varepsilon(0,800,1,600,2,400$ and 3,200). The treatments were repeated at least 3 times independently.

Determination of lactate dehydrogenase ( $L D H)$. In an attempt to properly simulate a physiological mechanical stimulation, LDH production of hBMSCs and hASCs was measured under different mechanical stimulation conditions and at different time points. LDH is a cytosolic enzyme which is released upon cell lysis. LDH production of hBMSCs and hASCs stimulated by CTS at magnitudes ranging from 0 to 3,200 $\mu \varepsilon$ was measured 1 day after cell culture. Subseqeently, CTS at a magnitude of 2,400 $\mu \varepsilon$ was applied to hBMSCs and hASCs. The LDH production of hBMSCs and hASCs was measured 1, 2, 4, 8, 12 and 14 days after cell culture. The LDH activity was measured using an LDH cytotoxicity detection kit. In brief, $150 \mu \mathrm{l}$ working solution and $50 \mu \mathrm{l}$ medium was mixed and then cultured in the dark at room temperature for $30 \mathrm{~min}$. Then the reaction was stopped by adding $1 \mathrm{~N} \mathrm{HCl}$, after which the absorbance at a wavelength of $570 \mathrm{~nm}$ was read. The results were expressed as arbitrary units and were adjusted to the unstimulated CTS control values.

In vitro osteogenesis under CTS and static controls. Cells were seeded on a polyethylene plastic dish at a density of $1.5 \times 10^{5}$ cells/plate in the above-mentioned osteogenic 

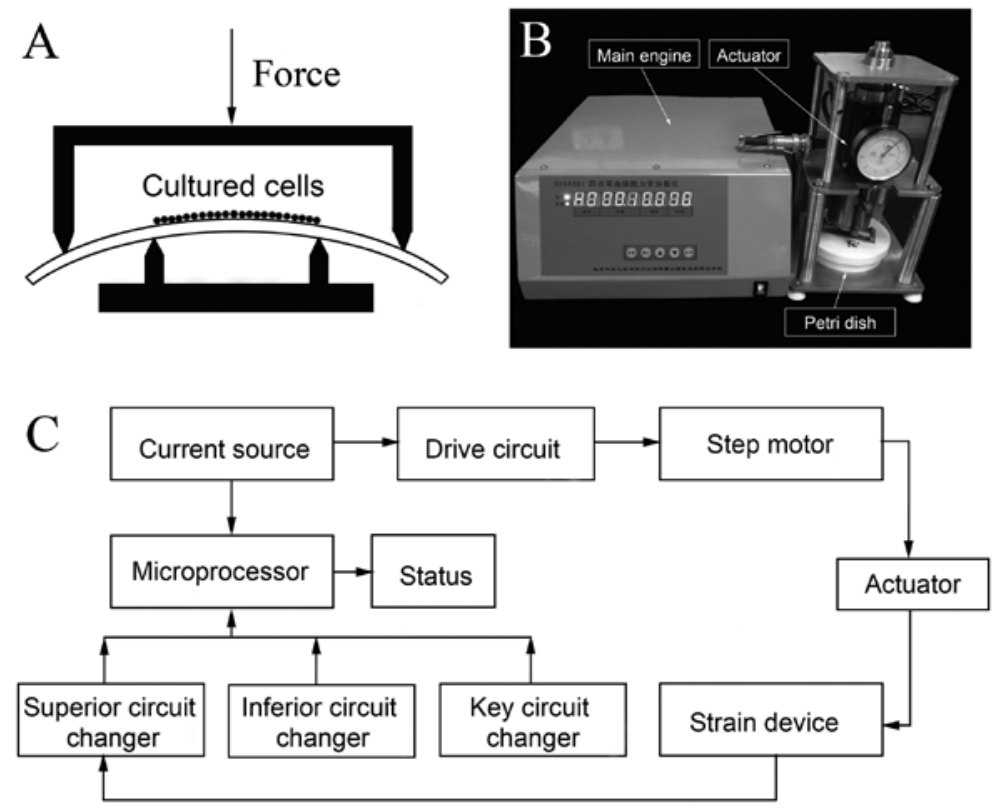

Figure 1. (A) A diagrammatic sketch of the CTS device. (B) The 4-point bending device consisted of 3 main parts: the main engine, the actuator and the cell culture Petri dish. (C) The main working process.

differentiation medium. When cells reached $80-90 \%$ confluence, they were subjected to the cyclic mechanical tensile stretch. The strain magnitude was $2,400 \mu \varepsilon$, frequency was $1 \mathrm{~Hz}$, with a 15 -sec rest after every 5 -sec working duration for a total of $2 \mathrm{~h} /$ day. The complete osteogenic differentiation process lasted for 14 days. The stimulated group consisted of cells cultured in osteogenic differentiation medium under CTS, whereas the unstimulated group consisted of cells that were cultured in the same differentiation medium but without exposure to CTS. The cell culture medium was replaced every 2 days.

Quantitative measurement of ALP activity and mineralization. Measurement of the ALP activity was performed 4 and 7 days after cell culture. ALP activity was measured by detecting the concentration of p-nitrophenol phosphate substrate. Cultured cells were lysed, and cell lysates were mixed with alkaline buffer for $\sim 15 \mathrm{~min}$. Then, ALP substrate was added to the mixture for another $30 \mathrm{~min}$, and finally the reaction was stopped by adding $0.05 \mathrm{~N} \mathrm{NaOH}$. The absorbance was read at a wavelength of $405 \mathrm{~nm}$, and the concentration was measured using a standard curve. Finally, the ALP activity was normalized to cellular protein concentration. Cellular protein concentration was detected as described by Lowry et al (17). Values are expressed as fold change over the static control, which were ASCs unstimulated by CTS for 4 days.

Measurement of mineralization (extracellular calcium deposition) was performed 10 and 14 days after the CTS. Cultured cells were washed twice with PBS and incubated in ethyl alcohol for $10 \mathrm{~min}$. Then, cells were washed with PBS another 3 times and stained with alizarin red to stain the extracellular calcium depositions. Nonspecific stained cells were washed with PBS for $15 \mathrm{~min}$. Extracellular calcium deposition was extracted using $10 \%(\mathrm{w} / \mathrm{v})$ cetylpyridinium chloride in $10 \mathrm{mM}$ sodium phosphate ( $\mathrm{pH}$ 7.0) for quantifica- tion. Absorbance at a wavelength of $562 \mathrm{~nm}$ was read and the concentration was determined using a standard curve. Values are expressed as fold change over the control, which were ASCs unstimulated by CTS for 10 days.

Real-time quantitative PCR analysis. Five and ten days after cell culture, total RNA was isolated with TRIzol reagent and used to synthesize cDNA with the Super-Script II cDNA synthesis kit (all were from Invitrogen Life Technologies). The osteogenic differentiation markers were assessed by quantitative real-time PCR using the SYBR-Green Master mix (ABI). Glyceraldehyde phosphate dehydrogenase (GAPDH) was selected as an internal control. The primer information is as follows: Runx2 (accession no. NM_001024630F) forward, CCAGATGGGACTGTGGTTACTG and reverse, TTCCGGA GCTCAGCAGAATAA; BMP-2 (accession no. NM_001200F) forward, GCCCTTTTCCTCTGGCTGAT and reverse, TTG ACCAACGTCTGAACAATGG; ALP (accession no. NM_013059) forward, CCTAGACACAAGCACTCCCACTA and reverse, GTCAGTCAGGTTGTTCCGATTC; OC (accession no. NM_199173F) forward, TGTGAGCTCAATCCGGA CTGT and reverse, CCGATAGGCCTCCTGAAAGC; GAPDH (accession no. NM_017008) forward, TATGACTCTACCCAC GGCAAGT and reverse, ATACTCAGCACCAGCATCACC.

Statistical analysis. All experiments were repeated at least 3 times independently. All the data are presented as mean \pm SD and analyzed with SPSS 13.0. The significance of difference was determined using the Student's t-test and analysis of variance (ANOVA); differences with $\mathrm{P}<0.05$ were considered significant.

\section{Results}

Morphology, surface antigen profiles and multi-differentiation ability of hBMSCs and hASCs. HBMSCs and hASCs 


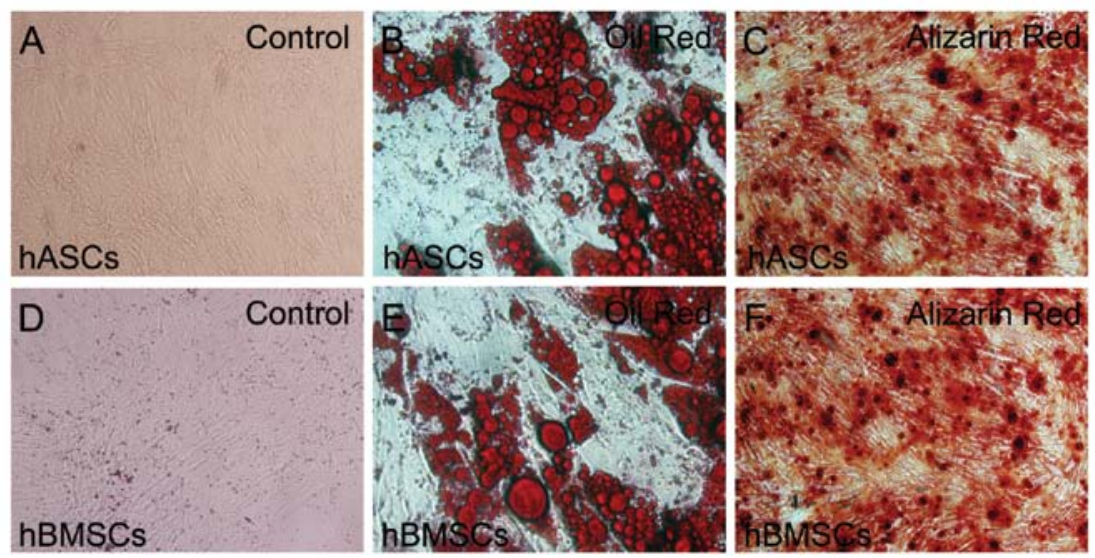

Figure 2. (A and D) Isolated ASCs and BMSCs showed a flat and fibroblast-like shape by passage 3. Both hASCs and hBMSCs could differentiate down (B and $\mathrm{E}$ ) an adipogenic lineage and ( $\mathrm{C}$ and $\mathrm{F})$ an osteogenic lineage under corresponding stimulation conditions.

\section{A}
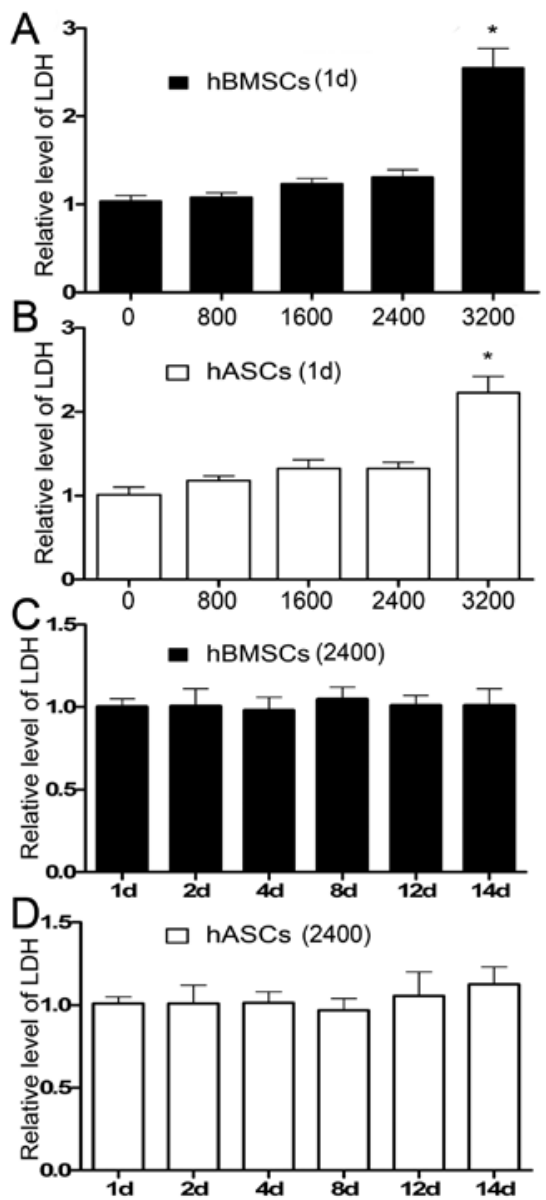

Figure 3. LDH production of hBMSCs and hASCs was measured under different strain magnitudes ( 0 to 3,200 $\mu \varepsilon$ ) and at different time points (1-14 days). (A and B) LDH production at a strain magnitude of 3,200 $\mu \varepsilon$ was significantly higher than that at other strain magnitudes 1 day after cell culture. (C and D) LDH production of hBMSCs and hASCs at a strain magnitude of $2,400 \mu \varepsilon$ showed no difference throughout the entire culture process $\left({ }^{*} \mathrm{P}<0.05\right)$.

passaged 3 times generally showed a homogeneous population containing flat and fibroblast-like cells (Fig. 2A and D). Flow cytometry results showed that both hBMSCs and hASCs positively expressed CD29 $(95.56 \pm 1.4 \%, 94.23 \pm 2.5 \%)$, CD44
$(94.65 \pm 2.2 \%, 96.10 \pm 1.9 \%)$, CD105 $(97.24 \pm 1.7 \%, 95.54 \pm 1.5 \%)$ and negatively expressed leukocyte common antigen CD45 $(6.7 \pm 0.8 \%, 5.4 \pm 1.2 \%)$, which was consistent with previous studies $(20,21)$.

To test the multi-differentiation ability of hBMSCs and hASCs, adipogenic differentiation and osteogenic differentiation assays were performed. Two weeks after adipogenesis, lipid droplets were clearly detected in both hBMSCs and hASCs, as demonstrated by oil red $\mathrm{O}$ staining (Fig. 2B and E). Two weeks after osteogenesis, both hBMSCs and hASCs were positively stained with alizarin red, and obvious calcification nodules were noted in both groups (Fig. 2C and F). Results of the flow cytometry and multi-differentiation test showed that both hBMSCs and hASCs were successfully isolated. Results also revealed that the surface antigen expression profiles and multi-differentiation capacity of hBMSCs and hASCs were similar to each other.

Effect of CTS on the survival of cultured cells. The LDH production of hBMSCs and hASCs measured 1 day after cell culture increased as the strain magnitude increased. The only exception was that the LDH production in the hASC group stimulated at a strain of $2,400 \mu \varepsilon$ was slightly lower than that at 1,600 $\mu \varepsilon$. There was no significant difference in LDH production 1 day after cell culture when the strain magnitude was $\leq 2,400 \mu \varepsilon$. Both hBMSCs and hASCs expressed a higher LDH production when the strain magnitude changed to $3,200 \mu \varepsilon$, which implied that a strain of 3,200 $\mu \varepsilon$ was not conducive to cell survival and should not be chosen as an ideal strain magnitude (Fig. 3A and B). The LDH production level was normalized to the level measured 1 day after cell culture of the unstimulated group.

A strain magnitude of $2,400 \mu \varepsilon$ was then applied to both hBMSCs and hASCs. The LDH production in both groups was then measured 1, 2, 4, 8, 12 and 14 days after cell culture. The LDH production level was normalized to the level measured 1 day after cell culture. Results showed that LDH production in both types of celsl remained practically equal to each other with no statistical difference (Fig. 3C and D). Based on these data, a strain at 2,400 $\mu \varepsilon$ was chosen to simulate the physiological mechanical stimulus in this study. 

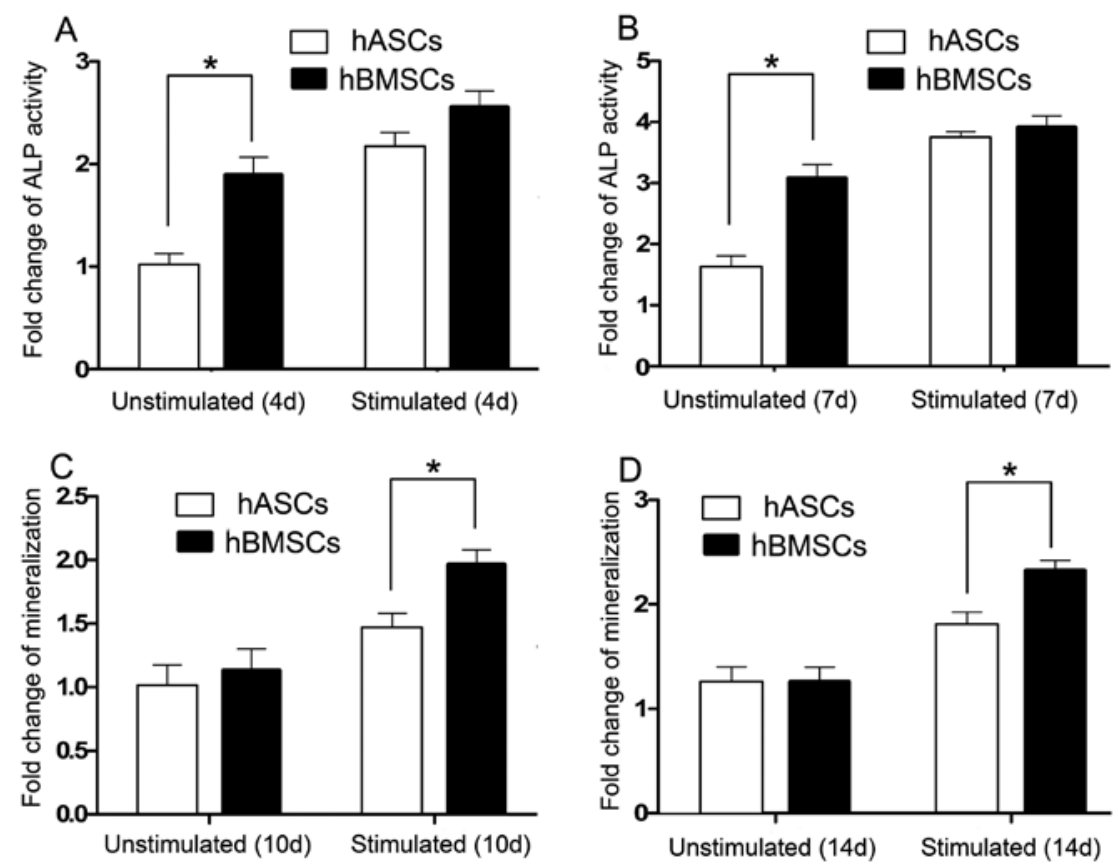

Figure 4. (A and B) Quantitative measurement of ALP activity in the hBMSCs and hASCs in the CTS-stimulated groups and CTS-unstimulated controls on days 4 and 7. (C and D) Quantitative measurement of mineralization as demonstrated by extracellular calcium deposition of the hBMSCs and hASCs in the CTS-stimulated groups and CTS-unstimulated controls on days 10 and $14\left({ }^{*} \mathrm{P}<0.05\right)$.
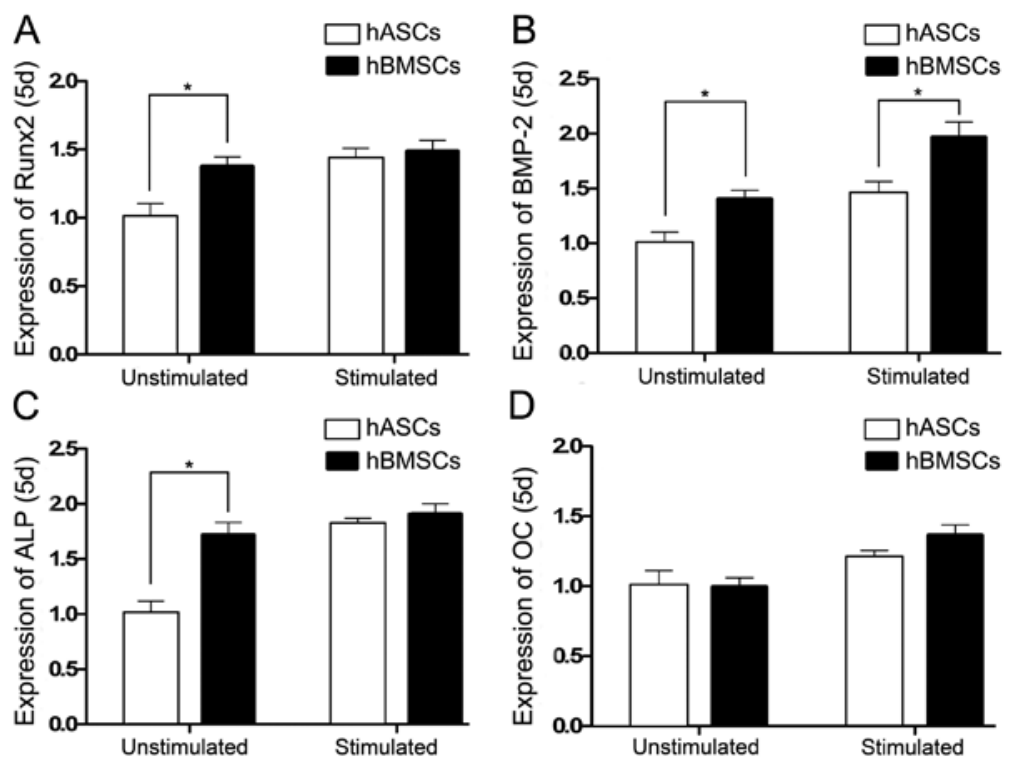

Figure 5. Real-time PCR analysis of hBMSCs and hASCs in the CTS-stimulated groups and CTS-unstimulated controls on day 5. (A) Runx2, (B) BMP-2, (C) ALP and (D) OC were selected as the osteogenic differentiation-related genes ("P<0.05).

Quantitative measurement of ALP activity. Quantitative measurement of ALP activity was performed 4 and 7 days after cell culture. Results showed that ALP activity of both hBMSCs and hASCs in the CTS-stimulated groups and CTS-unstimulated groups increased with time (Fig. 4A and B). The ALP activity of hBMSCs and hASCs unstimulated by CTS on days 4 and 7 showed a significant difference. However, when stimulated by CTS, the ALP activity in both the hBMSCs and hASCs increased and exhibited no difference on days 4 and 7 (Fig. 4A and B).
Quantitative measurement of mineralization. The mineralization, which was assessed by measuring extracellular matrix calcium depositions, occurred 10 and 14 days after cell culture. Results showed that both the extracellular matrix calcium deposition of hBMSCs and hASCs increased with time. Mineralization of hBMSCs and hASCs as represented by calcium depositions showed no difference in the CTS-unstimulated groups on days 10 and 14. When stimulated by CTS, mineralization of hBMSCs and hASCs increased and a significant difference was noted (Fig. 4C and D). 

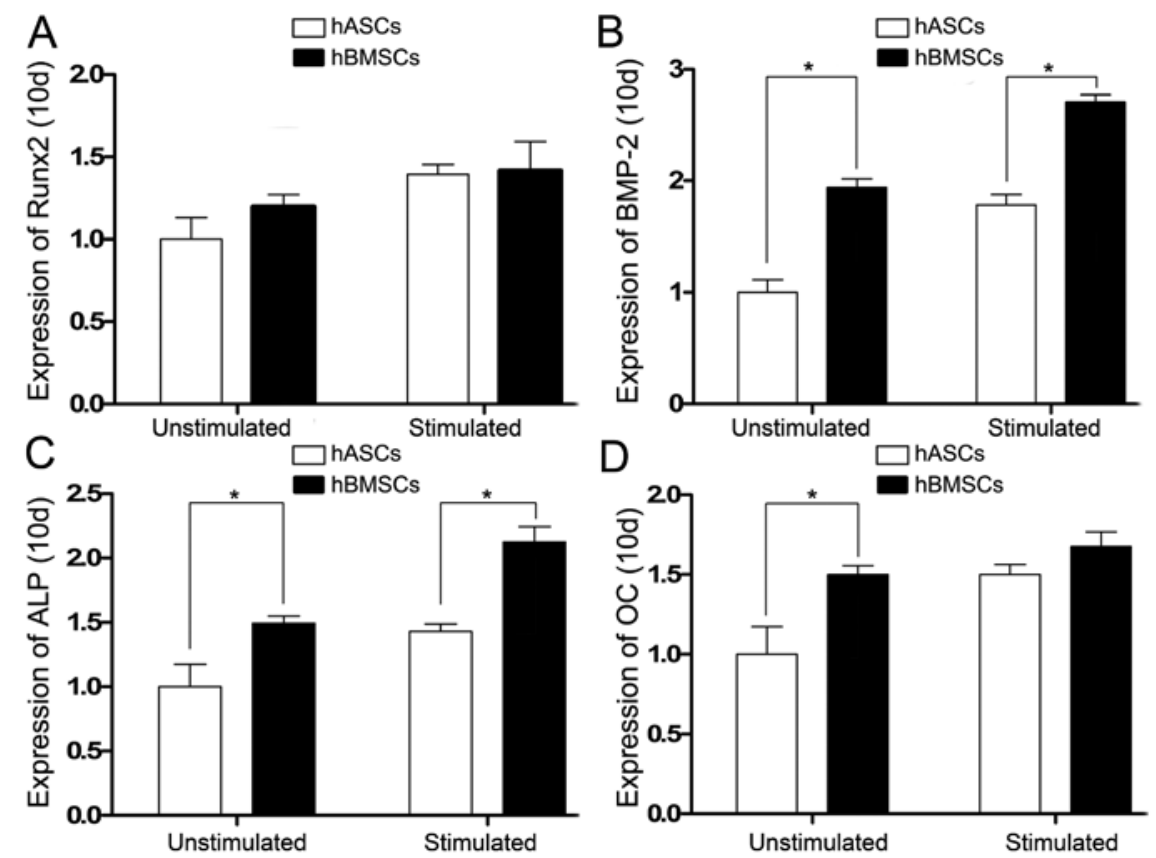

Figure 6. Real-time PCR analysis of hBMSCs and hASCs in the CTS-stimulated group and CTS-unstimulated controls at Day 10. (A) Runx2, (B) BMP-2, (C) ALP and (D) OC were selected as the osteogenic differentiation related genes ( $\mathrm{P}<0.05)$.

Osteogenic differentiation-specific mRNA expression profiles with and without exposure to CTS. We selected Runx2, bone morphogenetic protein-2 (BMP-2), ALP and osteocalcin (OC) as osteogenic differentiation-specific genes. The real-time PCR analysis was performed 5 and 10 days after cell culture.

Runx 2 gene expression in the hBMSCs and hASCs unstimulated by CTS on day 5 showed a significant difference. When stimulated by CTS, Runx 2 gene expression in both the hBMSCs and hASCs increased but the difference was not significant (Fig. 5A). Expression of the BMP-2 gene in the hBMSCs and hASCs in the CTS-unstimulated and stimulated groups on day 5 showed a significant difference, and the BMP-2 gene expression was increased when stimulated by CTS (Fig. 5B). ALP gene expression in the hBMSCs and hASCs were increased when stimulated by CTS. The ALP gene expression in the CTS-unstimulated hBMSCs and hASCs showed a significant difference, while no difference was noted in the CTS-stimulated groups (Fig. 5C). OC gene expression in the hBMSCs and hASCs in both the unstimulated and stimulated groups showed no significant difference on day 5. When stimulated with CTS, the OC expression of hBMSCs and hASCs demonstrated no increase (Fig. 5D).

Expression of the osteogenic differentiation-specific genes was also analyzed 10 days after cell culture. Runx 2 gene expression on day 10 in the hBMSCs and hASCs unstimulated and stimulated by CTS showed no statistical difference. When stimulated by CTS, Runx 2 expression in both the hBMSCs and hASCs increased (Fig. 6A). BMP-2 and ALP gene expression levels on day 10 in the hBMSCs were greater than levels of the hASCs in the CTS-unstimulated and stimulated groups and statistical differences were noted. When stimulated by CTS, BMP-2 and ALP expression in the hBMSCs and hASCs increased (Fig. 6B and C). OC gene expression on day 10 in the hBMSCs and hASCs unstimulated by CTS showed a statistical difference, but there was no statistical difference in the CTS-stimulated groups. When stimulated by CTS, OC expression in the hBMSCs and hASCs was increased (Fig. 6D).

\section{Discussion}

Bone tissue engineering has been developed in order to treat skeletal defects and other related clinical problems (18). With the progress of stem cell research, BMSCs have been widely utilized in bone tissue engineering. However, aspiration of bone marrow is painful and the yield in the cell concentration is relatively low. More recently, researchers have found that ASCs can also differentiate into mesodermal lineages such as chondrocytes and osteoblasts. The isolation of ASCs causes little harm to an organism and yields a large number of cells.

Numerous studies have compared the osteogenic differentiation capacity of BMSCs and ASCs cultured in static conditions without exposure to mechanical stimuli, yet the results have been inconsistent. Some researchers have reported that BMSCs possess an osteogenic differentiation capacity similar to that of ASCs $(8,9)$, while others have demonstrated that BMSCs possess a stronger osteogenic differentiation capacity than ASCs (10). A recent study conducted by Vishnubalaji et al (11) concluded that quantitative analysis is quite important in comparing the osteogenic potential of BMSCs and ASCs.

BMSCs and ASCs are not only sensitive to chemical but also mechanical stimuli. Numerous studies have shown that mechanical stimuli promote the development and function of engineered bone tissues (19). BMSCs and ASCs have both been widely used in the field of functional bone tissue engineering which includes mechanical stimuli. However, to our knowledge, there have been no studies aiming to compare the 
osteogenic differentiation capacity of hBMSCs and hASCs in response to mechanical stimuli.

In this study, BMSCs and ASCs were isolated from the same volunteers to eliminate differences caused by age and gender. Isolated BMSCs and ASCs were then tested for their multi-differentiation ability and surface antigen expression profiles. Results showed that both BMSCs and ASCs could differentiate along osteogenic and adipogenic pathways, formation of calcium nodules was observed in both groups undergoing osteogenesis and lipid droplets were also clearly detected in both groups undergoing adipogenesis. Flow results showed that both BMSCs and ASCs positively expressed CD29, CD44, CD105 and negatively expressed CD45; the flow results were consistent with previous studies $(20,21)$.

Previous studies have shown that excessive mechanical stress results in an elevated production of LDH (22). In an attempt to simulate physiological mechanical CTS but not excessive tensile stretch, the cultured cells were tested for the LDH production under different stretching magnitudes and time durations. Our study showed that a strain magnitude of $2,400 \mu \varepsilon$ is an effective physiological cyclic tensile strain. Isolated cells were cultured in the osteogenic differentiation medium with and without exposure to CTS.

ALP activity was quantified 4 and 7 days after cell culture. Our results showed that both hBMSCs and hASCs were sensitive to CTS, and the ALP activity of hBMSCs and hASCs increased when stimulated by CTS. The ALP activity of hBMSCs and hASCs in the unstimulated groups on days 4 and 7 was significantly different. When stimulated by CTS, the ALP activity increased in both cases, but no significant difference was noted.

The mineralization of the hBMSCs and hASCs was detected by extracellular matrix calcium deposition. Results showed that mineralization of hBMSCs and hASCs increased when stimulated by CTS. The mineralization of hBMSCs and hASCs showed no difference on days 10 and 14 in the unstimulated groups and showed a significant difference in the stimulated groups on days 10 and 14 .

Real-time PCR analysis was performed 5 and 10 days after cell culture. Runx2, a master gene that controls osteogenic differentiation (23), exhibited a difference in expression in the unstimulated groups of hBMSCs and hASCs 5 days after cell culture. When stimulated by CTS, the Runx 2 expression increased and showed no difference between the hBMSCs and hASCs. However, Runx 2 expression in the hBMSCs and hASCs measured 10 days after cell culture in the CTS stimulated and unstimulated groups showed no significant difference.

BMP-2 is a low-molecular-weight glycoprotein which functions as a morphogen and belongs to the transforming growth factor- $\beta$ (24). The gene expression of BMP-2 was also analyzed in this study. Our study showed that BMP-2 gene expression was elevated in the CTS-stimulated hBMSC and hASC groups when compared to that in the CTS-unstimulated hBMSCs and hASCs 5 and 10 days after cell culture. The BMP-2 gene expression of hBMSCs was greater than that in the hASCs both in the CTS-stimulated and unstimulated groups 5 and 10 days after cell culture. ALP gene expression of hBMSCs in the CTS-unstimulated group was greater than that of hASCs in the CTS-unstimulated group 5 and 10 days after cell culture, while ALP gene expression of hBMSCs in the CTS-stimulated group showed a similar expression level to that of hASCs in the CTS-stimulated group 5 days after cell culture. Additionally, the ALP gene expression of hBMSCs in the CTS-stimulated group was greater than that of the hASCs in the CTS-stimulated group 10 days after cell culture.

Finally, gene expression of OC was measured. OC is only secreted by osteoblasts and is commonly used as a marker of osteoblastic differentiation in the late stages of differentiation (25). OC expression in the hBMSCs and hASCs stimulated and unstimulated by CTS 5 days after cell culture showed no difference, and CTS did not promote OC expression in the hBMSCs and hASCs 5 days after cell culture. However, OC expression in the hBMSCs and hASCs unstimulated by CTS 10 days after cell culture showed a difference, and this difference was eliminated by the application of CTS.

In summary, this study compared the osteogenic differentiation capacity of hBMSCs and hASCs in both CTS-stimulated and unstimulated conditions. Our results revealed that CTS promoted the osteogenic differentiation of both hBMSCs and hASCs. The early-phase osteogenic differentiation capacity of hBMSCs stimulated by CTS was similar to that of hASCs stimulated by CTS as demonstrated by ALP activity measurement and RT-PCR analysis. The late-phase osteogenic differentiation capacity of hBMSCs stimulated by CTS was superior to that of hASCs stimulated by CTS as shown by mineralization measurement and RT-PCR. This study highlights the important role that mechanical stimuli play in functional bone tissue engineering and also provides critical information to the fields of functional bone tissue engineering and regenerative medicine.

\section{Acknowledgements}

This study was supported by grants from the National Natural Science Foundation of China (no. 31070831).

\section{References}

1. Potier E, Noailly $\mathrm{J}$ and Ito K: Directing bone marrow-derived stromal cell function with mechanics. J Biomech 43: 807-817, 2010.

2. Xiao C, Zhou H, Ge S, et al: Repair of orbital wall defects using biocoral scaffolds combined with bone marrow stem cells enhanced by human bone morphogenetic protein- 2 in a canine model. Int J Mol Med 26: 517-525, 2010.

3. Arita NA, Pelaez D and Cheung HS: Activation of the extracellular signal-regulated kinases 1 and 2 (ERK1/2) is needed for the TGFbeta-induced chondrogenic and osteogenic differentiation of mesenchymal stem cells. Biochem Biophys Res Commun 405: 564-569, 2011.

4. Bodle JC, Hanson AD and Loboa EG: Adipose-derived stem cells in functional bone tissue engineering: lessons from bone mechanobiology. Tissue Eng Part B Rev 17: 195-211, 2011.

5. Panetta NJ, Gupta DM, Lee JK, Wan DC, Commons GW and Longaker MT: Human adipose-derived stromal cells respond to and elaborate bone morphogenetic protein-2 during in vitro osteogenic differentiation. Plast Reconstr Surg 125: 483-493, 2010.

6. Tapp H, Hanley EJ, Patt JC and Gruber HE: Adipose-derived stem cells: characterization and current application in orthopaedic tissue repair. Exp Biol Med (Maywood) 234: 1-9, 2009.

7. Liu G, Cheng Y, Guo S, et al: Transplantation of adipose-derived stem cells for peripheral nerve repair. Int J Mol Med 28: 565-572, 2011.

8. De Ugarte DA, Morizono K, Elbarbary A, et al: Comparison of multi-lineage cells from human adipose tissue and bone marrow. Cells Tissues Organs 174: 101-109, 2003. 
9. Hattori H, Sato M, Masuoka K, et al: Osteogenic potential of human adipose tissue-derived stromal cells as an alternative stem cell source. Cells Tissues Organs 178: 2-12, 2004.

10. Im GI, Shin YW and Lee KB: Do adipose tissue-derived mesenchymal stem cells have the same osteogenic and chondrogenic potential as bone marrow-derived cells? Osteoarthritis Cartilage 13: 845-853, 2005.

11. Vishnubalaji R, Al-Nbaheen M, Kadalmani B, Aldahmash A and Ramesh T: Comparative investigation of the differentiation capability of bone-marrow- and adipose-derived mesenchymal stem cells by qualitative and quantitative analysis. Cell Tissue Res 347: 419-427, 2012.

12. Ghazanfari S, Tafazzoli-Shadpour M and Shokrgozar MA: Effects of cyclic stretch on proliferation of mesenchymal stem cells and their differentiation to smooth muscle cells. Biochem Biophys Res Commun 388: 601-605, 2009.

13. Colazzo F, Sarathchandra P, Smolenski RT, et al: Extracellular matrix production by adipose-derived stem cells: implications for heart valve tissue engineering. Biomaterials 32: 119-127, 2011.

14. Sarraf CE, Otto WR and Eastwood M: In vitro mesenchymal stem cell differentiation after mechanical stimulation. Cell Prolif 44: 99-108, 2011.

15. Sumanasinghe RD, Bernacki SH and Loboa EG: Osteogenic differentiation of human mesenchymal stem cells in collagen matrices: effect of uniaxial cyclic tensile strain on bone morphogenetic protein (BMP-2) mRNA expression. Tissue Eng 12: 3459-3465, 2006

16. Goli-Malekabadi Z, Tafazzoli-Shadpour M, Rabbani M and Janmaleki M: Effect of uniaxial stretch on morphology and cytoskeleton of human mesenchymal stem cells: static vs. dynamic loading. Biomed Tech (Berl) 56: 259-265, 2011.
17. Lowry OH, Rosebrough NJ, Farr AL and Randall RJ: Protein measurement with the Folin phenol reagent. J Biol Chem 193: 265-275, 1951.

18. El HA and Cartmell SH: Bioreactors for bone tissue engineering. Proc Inst Mech Eng H 224: 1523-1532, 2010.

19. Tirkkonen L, Halonen H, Hyttinen J, et al: The effects of vibration loading on adipose stem cell number, viability and differentiation towards bone-forming cells. J R Soc Interface 8: 1736-1747, 2011.

20. Dominici M, Le Blanc K, Mueller I, et al: Minimal criteria for defining multipotent mesenchymal stromal cells. The International Society for Cellular Therapy position statement. Cytotherapy 8: 315-317, 2006.

21. Mitchell JB, McIntosh K, Zvonic S, et al: Immunophenotype of human adipose-derived cells: temporal changes in stromalassociated and stem cell-associated markers. Stem Cells 24: 376-385, 2006.

22. Kang MN, Yoon HH, Seo YK and Park JK: Effect of mechanical stimulation on the differentiation of cord stem cells. Connect Tissue Res 53: 149-159, 2012.

23. Otto F, Thornell AP, Crompton T, et al: Cbfa1, a candidate gene for cleidocranial dysplasia syndrome, is essential for osteoblast differentiation and bone development. Cell 89: 765-771, 1997.

24. Colnot C: Cell sources for bone tissue engineering: insights from basic science. Tissue Eng Part B Rev 17: 449-457, 2011.

25. Franceschi RT and Iyer BS: Relationship between collagen synthesis and expression of the osteoblast phenotype in MC3T3-E1 cells. J Bone Miner Res 7: 235-246, 1992. 